\title{
Analysis of Drug-Related Problems Identified by Future Pharmacists in Medical Wards of Tertiary Hospitals in the State of Pahang, Malaysia
}

\author{
Mohamed Hassan Elnaem*, Nor llyani Mohamed Nazar, Norny Syafinaz Ab Rahman, Shazia Jamshed \\ Department of Pharmacy Practice, Faculty of Pharmacy, International Islamic University Malaysia, Kuantan, Pahang, MALAYSIA.
}

\begin{abstract}
Objective: Clinical attachments undertaken by pharmacy students in Malaysia are one of the important platforms in order to prepare future candidates for patient-centred pharmacy practice. The lack of data regarding the identification of Drug-related problems (DRPs) and the suggestion of relevant interventions by final year pharmacy students has directed the current research to describe pharmacy students' abilities in identifying DRPs in their final year clinical placements. Methods: As a descriptive cross-sectional study, it involved the analysis of the clinical case reports of final year pharmacy students during their six-week clinical attachments between February and April 2017 across three different tertiary hospitals in the state of Pahang, Malaysia. A six-member team, who had received the required training by the study's principal researcher, performed the data extraction from the clinical attachment reports. Results: A total of 385 clinical case reports, identified 880 DRPs. The two most common types of identified DRPs were the untreated condition (17.4\%), followed by inappropriate drug selection (16.3\%). Nearly $47.7 \%$ of total detected DRPs were related to the indication and efficacy domains of therapy assessment. Additionally, there was a positive correlation found between the number of
\end{abstract}

underlying diseases and the number of identified DRP, $r_{s}(372)=.14, p=.007$. Conclusion: During the final year clinical attachments, the majority of the students were able to identify a considerable number of DRPs in the hospital setting under the supervision of their preceptors. The characterisation of the identified DRPs could be instrumental in refining the experiential clinical learning among future pharmacy practitioners.

Key words: Drug-related problem, Experiential learning, Pharmacy students, Clinical attachment.

\section{Correspondence}

Dr. Mohamed Hassan Elnaem, BPharm, PharmD, BCPS, Lecturer, Department of Pharmacy Practice. Faculty of Pharmacy, International Islamic University Malaysia Jalan Sultan Ahmad Shah, 25200 Kuantan, Pahang, MALAYSIA.

Phone: +60 193944726

Email: drmelnaem@gmail.com; drmelnaem@iium.edu.my

DOI: 10.5530/jyp.2019.11.16

\section{INTRODUCTION}

Over the past few decades, the pharmacist's role has increasingly become important given their participation and contribution in health care teams and towards assuring appropriate medication therapy management for patients. ${ }^{1}$ The collaborative healthcare model has also involved pharmacists in utilising their medication management knowledge and skills to support the physician's therapy strategies which have shown to improve the patients' adherence and satisfaction with the recommended treatment plans. ${ }^{2}$ Notwithstanding, the involvement and contribution of pharmacists, as healthcare professionals, has remarkably helped to reduce medication-related hospital readmissions linked to financial savings in the health care system. ${ }^{3-4}$

Drug-related problems (DRP) can be defined broadly as involving all conditions related to drug therapy that may actually or potentially be interfering with the attainment of optimal treatment outcomes. ${ }^{5}$ DRPs regarding the choice and use of drugs including interactions are relatively common among hospitalised patients which if not managed correctly may result in unwanted clinical and economic consequences. ${ }^{6}$ Indeed, within various clinical specialities, the involvement of clinical pharmacists in patient care was found to positively enhance the efficiency of the overall process through providing medication reconciliation services to minimise the incidence and reoccurrence of DRPs and related consequences. ${ }^{7}$ Moreover, the inclusion of clinical pharmacists in dispensing medical practices in Scotland, in the pharmaceutical care of patients has helped to optimise the impact of medication treatment programs in both the quantitative and qualitative service aspects. ${ }^{8}$ Notwithstanding, community pharmacists could equally beneficial to reduce the burden of potential DRPs upon hospital discharge patients provided they be provided access to hospital patient discharge summaries. ${ }^{9}$

There are numerous DRP terminologies and classifications found in the literature. However, there are only a few classifications that have been validated in clinical practice. ${ }^{10}$ Also, various DRP classification systems have employed different structures regarding DRP definitions, probable causes and proposed interventions. ${ }^{11}$ A study by the Czech Republic to establish standards for clinical pharmacy services has shown that undertaking a systemic drug review at pre-set intervals was effectively able to identify DRP causes and relevant interventions across various clinical specialities. ${ }^{12}$ The common DRPs that have been identified in clinical practice include; improper drug use, inappropriate prescribing of medications, discrepancies between prescribed and actual regimens, drug interactions, poor adherence by patients, lack of patient monitoring and inadequate surveillance for adverse effects. ${ }^{13}$ Furthermore, DRP related interventions could be categorised into three main categories, namely; in the introduction, discontinuation or changing the currently prescribed medications. ${ }^{12}$

Clinical pharmacists in medication therapy management generally follow two approaches; a pro-active approach and a reactive approach. A pro-active approach involves the suggestion of interventions during prescribing or ordering medications. This approach has shown a higher

This is an open access article distributed under the terms of the Creative Commons Attribution-NonCommercial-ShareAlike 4.0 License, which allows others to remix, tweak, and build upon the work non-commercially, as long as the author is credited and the new creations are licensed under the identical terms. 
acceptance rate by physicians compared to the reactive approach, such as writing comments after prescribing the medication. ${ }^{14}$ The higher acceptance rate by physicians was also influenced by the presence of pharmacotherapy experts during ward rounds and through the prioritisation of most clinically relevant DRPs. ${ }^{15}$

The involvement of a clinical pharmacist has also been shown to have a positive impact on the clinical outcomes associated with lipid-lowering therapy used in the management of dyslipidaemia. ${ }^{16}$ Moreover, research conducted on the resolution of DRPs by adopting a team-based approach has shown that pharmacists had a significant impact on the optimisation and use of medications. ${ }^{17}$ It has further been reported that the involvement of pharmacists within a team environment along with different health care professionals, such as nurses and social workers, enables the pharmacists to focus more on prescribing-improvement interventions and to be able to communicate with physicians to resolve DRPs effectively. ${ }^{17}$

Clinical clerkships undertaken by pharmacy students in Malaysia are one of the essential platforms to prepare future practitioners for pharmaceutical care practices in hospitals and community settings. ${ }^{18}$ For example, the impending pharmacists are expected to develop and further enhance their competencies in teaming with other healthcare professionals, through collaborative clinical interactions during clinical clerkships. ${ }^{19}$ Self-directed learning during clinical clerkships is another important facet towards refining their competencies as future pharmacists which helps them in being recognised as 'pharmacotherapy experts' amongst different healthcare professionals. ${ }^{19}$

According to previous research, comparing various health professional students in their ability to identify and correct medication prescribing errors, pharmacy students showed significantly higher rates towards identifying prescribing error compared with medical and nursing students. ${ }^{20}$ Moreover, it has also been reported that providing pharmacy students with real-life examples, opportunities and experiences regarding medication reconciliation, history taking and medication chart reviews; has been shown to have a positive impact on their ability to identify and resolve DRPs with an overall acceptance rate exceeding $70 \%$ based on their interventions. ${ }^{21}$

The different aspects of experiential learning offered in pharmacy schools are fundamental in preparing future pharmacists for refining and exposure to evolving healthcare services. ${ }^{22}$ The capability of pharmacy students to identify and analyse DRPs in this current setting is an area of increasing investigation. Therefore, the current research aims to summarise the experiential learning aspects of final year pharmacy students' by describing the content of their clinical attachment reports generated through their placements in three different tertiary hospitals.

\section{METHODOLOGY}

\section{Study design}

As a descriptive cross-sectional study, the process involved the analysis of clinical attachment reports from final year pharmacy students for six-week duration from February to April 2017.

\section{Study setting}

The students were attached to three different tertiary hospitals in the state of Pahang, Malaysia. They were required to clerk two cases per week and rotated between departments on a weekly basis. They have to follow the patients' progress note during the hospital stay and to communicate regularly with healthcare practitioners and their preceptors.

\section{Study instrument and data collection}

In order to retrieve the information from the case reports, a data extraction form was designed in compliance with the clinical pharmacy report form officially used in government hospitals to describe and quantify pharmacistinitiated suggestions in order to optimise medication therapy. The form was adapted to retrieve all relevant information needed to describe any identified DRP comprising of four sections; A, B, C and D. Section A included the patient's demographic. Section B described the patient's medical history, specifically the drugs and disease status as well as the presence of any hypersensitivity. Section C comprised of the patient's laboratory investigations including; blood glucose level, blood pressure, serum cholesterol level, liver function test (ALT and AST level), creatinine clearance, blood count test (haematocrit and white blood cell) and serum uric acid level and sequentially comparing the values against their respective reference measures. Section $\mathrm{D}$ contained all the relevant areas related to the medical treatment that the patient was receiving in the ward, including final diagnosis, in-ward medications and DRGs encountered during the clerkship period, categorised based on the Hepler and Strand classification. ${ }^{10}$ The data collection process involved team of six members who had received relevant training by the principal researcher prior to the study.

\section{Statistical analysis}

All completed data collection forms were included in the data analysis. Descriptive statistics using frequencies and proportions were used to describe the number and types of identified DRPs during the final year of clinical clerkship. The factors associated with the type of DRP and type of in-ward medication were initially investigated with the Chi-square test of independence and the level of significance was set at $P<0.05$. Data were analysed using the Statistical Package for the Social Sciences (SPSS) v.22 software. (IBM SPSS Statistics, NC, USA).

\section{RESULTS}

\section{Patient's Demographic and Past Medical History}

There was a total of 385 clinical case reports included in the study. Overall, $40 \%$ of the clinical reports examined were of female patients. The findings showed that about $70 \%$ of the patients were aged 50 years or older and approximately $60 \%$ of patients were identified as being overweight. A considerable portion of patients admitted to the three hospitals had 3 or 2 concomitant diseases, comprising $25.4 \%$ and $20.9 \%$ of all patients, respectively. In contrast, a relatively small portion of patients did have any prior medical history (6.1\%). Figure 1 illustrates the percentage of the patient cohort according to the number of concomitant diseases.

The abnormalities were tracked from the investigated laboratory data in order to outline which parameters might deserve more focus towards understanding the clinical consequences. The abnormal values representing renal and blood pressure accounted for the highest recorded laboratory data in our study with more than $70 \%$ of the patients with recorded values above the normal range. Table 1 represents the overall recorded abnormal values of the laboratory data.

\section{In ward management}

Regarding the description of the individual use of each medication group, it was found that cardiovascular agents showed the highest percentage $(24.4 \%)$ from among the other agents followed by the antiinfective drugs (19.8\%) and gastrointestinal/ hepatobiliary drugs (16.2\%). Figure 2 displays the percentage of use of each class of medication among the selected sample. 


\section{Drug-related problem}

Overall, there were 880 identified drug-related problems (DRPs). It is important to acknowledge that the two most common types of DRPs encountered were related to the untreated condition (17.4\%), followed by improper drug selection (16.3\%). Figure 3 represents the overall percentage of individual types of DRPs that were identified in the sample. Furthermore, it was highlighted that the use of cardiovascular (CVS) and respiratory (Resp) drugs along with anti-infective agents (AIA) in an individual patient accounted for more than half $(60.9 \%)$ of the total medication combination used in the hospitals. Whereas, for the type of DRPs, the occurrence of both indication and efficacy related DRPs contributed the highest percentages $(47.7 \%)$ compared to other DRPs. Table 2 outlines the overall grouping of medications and DRP types.

\section{Association between underlying diseases, in-ward medications and DRP types}

A Kruskal-Wallis $\mathrm{H}$ test was carried out to determine if there were differences in the total number of identified DRPs between the different classes of in-ward medications. The distribution of DRP numbers was not similar for all groups and was assessed by visual inspection of a boxplot. The mean rank of the DRP numbers was not statistically, significantly different between different medication groups $x 2(5)=6.771, p=0.238$. Moreover, Goodman and Kruskal's $\lambda$ was run to determine whether the type of identified DRP could be better predicted by knowledge of the medication class prescribed for the patient. In this case, there was no statistically significant reduction in the proportions of errors due to the knowledge of the medication class given to an individual patient, $p=0.563$.

Finally, a Spearman's rank-order correlation was carried out to assess the relationship between the number of underlying medical conditions and the identified DRP number. Preliminary analysis showed the relationship to be monotonic, as assessed by visual inspection of a scatterplot. However, there was a positive correlation between some underlying diseases and some identified DRPs, $r_{s}(372)=.14, p=.007$.

\section{DISCUSSION}

The findings related to the type of DRPs showed that both the untreated condition and inappropriate drug choices were the two most frequently identified DRPs. In a similar study undertaken in the United States, it was highlighted that PharmD students identified the need for therapy and dose adjustment as being the highest recorded DRPs during their clinical placement. ${ }^{23}$ Correspondingly, a German study conducted to evaluate pharmaceutical care interventions suggested by clinical pharmacists reported that the main suggestions in over half of the subjects in the study were related to the addition, replacement or withdrawal of drugs. ${ }^{24}$ In contrast, pharmacy students in the study conducted by Armor and his colleagues reported that the incomplete medication list and the need for laboratory test were the two most common DRPs during their experiential clinical placement. ${ }^{21}$ Notwithstanding, in a local study aimed to investigate the type of DRPs among diabetic subjects with underlying hypertension, reported that the two most common DRPs were insufficient health awareness and inappropriate drug choices. ${ }^{25}$ The findings in our study were considered different from previous Swedish research that was carried out that revealed both inappropriate drug use and interactions were the most common DRPs identified by clinical pharmacists in a hospital setting. ${ }^{6}$ In a separate study undertaken in an Indian hospital setting, it identified the same findings through characterising both drug interactions and drug use problems as the most common identified DRPs. ${ }^{13}$ Overall, the knowledge of common identified DRPs in clinical practice could be of enormous

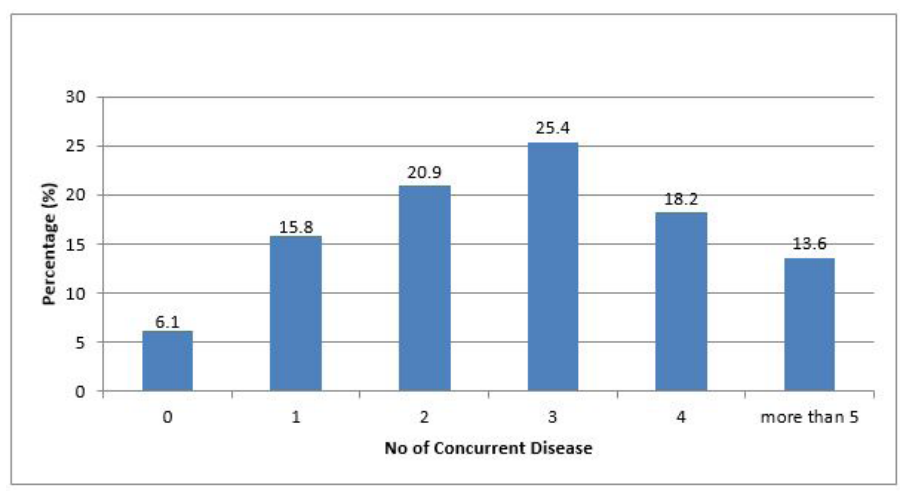

Figure 1: Number of concurrent diseases in the past medical history.

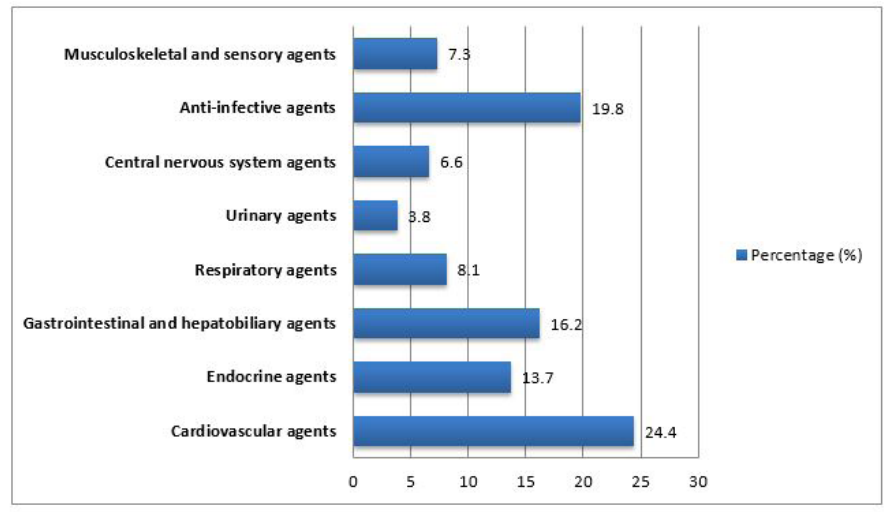

Figure 2: Class of Medications used in Ward Management.

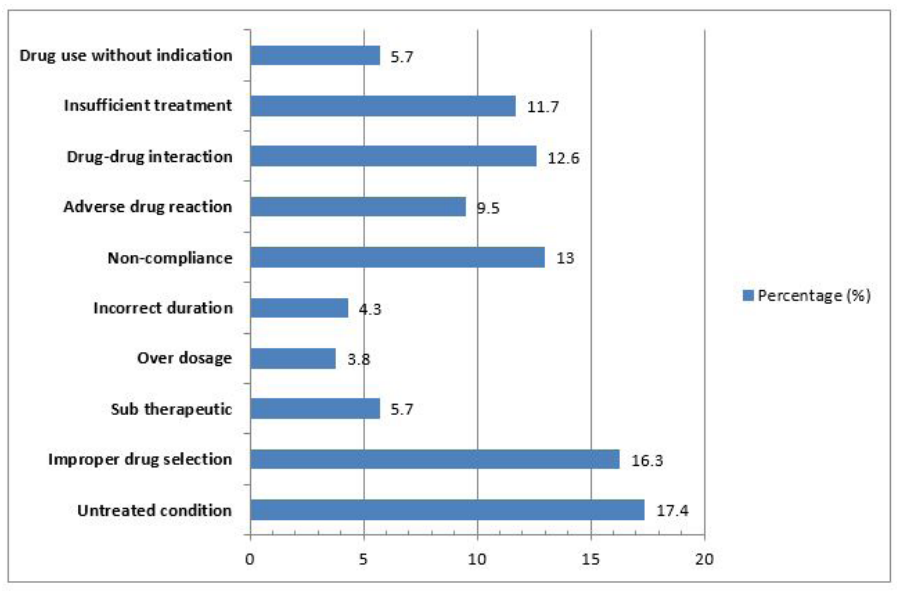

Figure 3: The individual percentage of Drug-Related Problems (DRPs).

benefit in designing training before the final year of experiential clinical education for pharmacy students.

Concerning the number of DRPs, this study highlighted the identification of 2.28 DRPs per patient which is lower than the number of DRPs per patient identified by clinical pharmacists in a study in internal medicine wards in a Swiss hospital setting. ${ }^{15}$ However, the reported findings in our study are considered relatively higher in comparison with previous studies which reported 1.29, 1.53 and 1.93 DRPs per patient, respectively. ${ }^{6,13,21}$ This shows that the frequency and type of identified DRPs may vary according to the setting. Accordingly, this variation may postulate some 
Table 1: Frequency and percentage of laboratory parameters abnormalities as per the clinical attachment reports.

\begin{tabular}{ccc}
\hline $\begin{array}{c}\text { Lab parameters } \\
\text { (Abnormal) }\end{array}$ & Frequency (N) & Percentage (\%) \\
\hline Random blood glucose & 142 & 36.6 \\
Blood pressure & 276 & 71.1 \\
Serum cholesterol level & 14 & 3.6 \\
ALT & 45 & 11.6 \\
AST & 52 & 13.4 \\
Creatinine clearance & 279 & 71.9 \\
Haematocrit level & 118 & 30.4 \\
White blood cell count & 193 & 41.7 \\
Serum Uric Acid & 61 & 15.7 \\
\hline
\end{tabular}

differences in the competency and knowledge of the clinical pharmacist or student pharmacists involved in the medication reviews.

With regards to the common drugs used in this study's setting, it was highlighted that cardiovascular drugs followed by anti-infective agents were the most commonly used treatments. This finding is similar to previous work that reported cardiovascular medications as being the most frequently prescribed treatment among hospitalised patients. ${ }^{6}$ Furthermore, a study conducted with a focus on patients with cardiovascular diseases, highlighted that one-third of the study sample had been identified with at least one DRP. ${ }^{26}$ The high prevalence of cardiovascular diseases and the frequent use of cardiovascular drugs underscores the need for proper training and preparation of future healthcare professionals in the cardiology clinical practice. ${ }^{27}$ The frequent use of anti-infective agents was implicated in the findings of our study having potential contributions towards the DRPs detected in our study's setting. In contrast, a study conducted in Lebanon reported anti-infective agents as being among the lowest medication class associated with DRPs. ${ }^{28}$

Furthermore, the work in our study showed that the type of DRPs encountered had no significant association with the type of medication. Furthermore, the findings in our study did not support the fact that certain medications could be associated with a higher DRP prevalence risk. This result did not resemble the previous research reports that highlighted the antihypertensive drugs, anticoagulants and corticosteroids, as being the most likely medication classes associated with DRPs, namely; drug interactions, dosage problems and contraindications. ${ }^{28}$ In a separate study by Viktil et al. it was revealed that polypharmacy is a relatively weak indicator in the assessment of DRPs. ${ }^{29}$ Similarly, the findings in our study did not support a link between some detected DRPs and the number of prescribed medications. On the other hand, our study highlighted a significant correlation between the number of detected DRPs and the number of patient's concomitant diseases. This result is in line with several published research papers that reported some underlying diseases as principal contributors towards the increased number of identified DRPs during hospitalisation. ${ }^{6}$ Also, our results seem to be in agreement with a previous study highlighting the high prevalence of DRPs among patients with at least two underlying diseases..$^{30}$ Importantly, it is also worthy to highlight that certain medical conditions were reported in the literature to be associated with the prevalence of DRPs that were commonly identified through clinical pharmacists' interventions. ${ }^{16,31}$
Table 2: Type of Medications and DRGs encountered during Patient Hospitalisation.

\begin{tabular}{lcc}
\hline \multicolumn{3}{c}{ Type of medications used in the ward } \\
\hline & Frequency & Percentage (\%) \\
\hline 1. CVS + Resp & 18 & 6.0 \\
2. CVS + Endo & 46 & 15.4 \\
3. CVS + Resp + AIA & 182 & 60.9 \\
4. CVS + Endo + GIT & 53 & 17.7 \\
& 299 & 100 \\
& Types of DRPs \\
1. Indication + Efficacy & 180 & \\
2. Indication + Safety & 71 & 18.7 \\
3. Indication + Compliance & 79 & 21.0 \\
4. Indication + Efficacy + Safety & 47 & 12.5 \\
& 377 & 100 \\
\hline
\end{tabular}

\section{LIMITATIONS}

The study has several limitations. First, the students' clinical attachment reports were considered as the primary source of information, which might subject our findings to reporting bias. However, the pharmacy preceptors were asked to evaluate the content of the reports and approve the DRPs identified by the student pharmacists to address this concern. Secondly, although training was provided to all student pharmacists who aided in the data collection, their clinical knowledge and experience varied to the extent of underreporting some potential DRPs. Lastly, this study did not manage to identify the specific type of drugs related to certain types of DRPs.

\section{CONCLUSION}

During the clinical clerkships, the final year pharmacy students were able to identify a significant number of DRPs in the hospital setting under the supervision of their preceptors. For further study, the characterisation of the identified DRPs may be beneficial towards improving experiential clinical pharmacy learning in the future.

\section{ACKNOWLEDGEMENT}

The research team would like to express their gratitude to all final year pharmacy students whose reports were analysed during the study. Additionally, we acknowledge the invaluable contribution of all students who were involved in the data collection. Furthermore, we truly appreciate the supportive role of the Faculty of Pharmacy in facilitating the study approval and data collection procedures. Finally, we are grateful to the support received under a grant (ID: RIGS17-122-0697) by the research management centre of the International Islamic University Malaysia.

\section{CONFLICT OF INTEREST}

The authors declare no conflict of interest.

\section{ABBREVIATIONS}

DRPs: Drug-related problems; ALT: Alanine Aminotransferase; AST: Aspartate Aminotransferase; SPSS: Statistical Package for the Social Sciences; CVS: Cardiovascular; Resp: Respiratory; AIA: Anti-infective agents. 


\section{REFERENCES}

1. Bush PW, Daniels R. Health Care Systems and Transitions of Care. N C Med J 2017;78(3):177-80.

2. Finley PR, Rens HR, Pont JT, Gess SL, Louie C, Bull SA, et al. Impact of a collaborative pharmacy practice model on the treatment of depression in primary care. Am J Heal Pharm. 2002;59(16):1518-26.

3. Blouin RA, Adams ML. The Role of the Pharmacist in Health Care. N C Med J. 2017;78(3):165-7.

4. Farley JF, Ferreri SP, Easter JC, McClurg MR. The North Carolina Experiment. N C Med J. 2017;78(3):186-90.

5. Krähenbühl-Melcher A, Schlienger R, Lampert M, Haschke M, Drewe J Krähenbühl S. Drug-Related Problems in Hospitals. Drug Saf. 2007;30(5):379-407.

6. Peterson C, Gustafsson M. Characterisation of Drug-Related Problems and Associated Factors at a Clinical Pharmacist Service-Naïve Hospital in Northern Sweden. Drugs - Real World Outcomes. 2017;4(2):97-107.

7. Francis J, Abraham S. Clinical pharmacists: Bridging the gap between patients and physicians. Saudi Pharm J. 2014;22(6):600-2.

8. Stewart D, Anthony B, Morrison C, MacRae Y, Dixon L, Friel E, et al. Evaluating pharmacist input into the pharmaceutical care of patients in dispensing medical practices in remote and rural areas of Scotland. Fam Pract. 2017;34(4):491-9.

9. Braund R, Coulter CV, Bodington AJ, Giles LM, Greig AM, Heaslip LJ, et al. Drug related problems identified by community pharmacists on hospital discharge prescriptions in New Zealand. Int J Clin Pharm. 2014;36(3):498-502.

10. Van Mil JWF, Westerlund LOT, Hersberger KE, Schaefer MA. Drug-Related Problem Classification Systems. Ann Pharmacother. 2004;38(5):859-67.

11. Pk A, Adepu R. Drug related problems: an over view of various classificaton systems. Asian J Pharm Clin Res. 2014;7(4):7-10.

12. Gregorová J, Rychlícková J, Šaloun J. Standardization of clinical pharmacist's activities: Methodology. Saudi Pharm J. 2017;25(6):927-33.

13. Sarfaraz M, Mathew B, Poudel S. Assessment of Drug Related Problems in a Tertiary Care Teaching Hospital, India. Asian J Pharm Clin Res. 2017;10(2):310-3.

14. Viktil KK, Blix HS. The impact of clinical pharmacists on drug-related problems and clinical outcomes. Basic Clin Pharmacol Toxicol. 2008;102(3):275-80.

15. Guignard B, Bonnabry P, Perrier A, Dayer P, Desmeules J, Samer CF. Drug-related problems identification in general internal medicine: The impact and role of the clinical pharmacist and pharmacologist. Eur J Intern Med. 2015;26(6):399-406.

16. Till TL, Voris JC, Horst JB. Assessment of Clinical Pharmacist Management of Lipid-Lowering Therapy in a Primary Care Setting. J Manag Care Pharm. 2003;9(3):269-73.

17. Renfro CP, Ferreri SP, Williams N, Clark C, Pfeiffenberger T. Description of drug therapy problem resolution in a statewide care management program. J Am Pharm Assoc. 2017;57(3):S289-S292.

18. Elnaem MH, Jamshed SQ, Elkalmi RM. The future of pharmaceutical care in Malaysia : Pharmacy students ' perspectives. Pharm Educ. 2017;17(1):215-22.

19. Jungnickel PW, Kelley KW, Hammer DP, Haines ST, Marlowe KF. Addressing competencies for the future in the professional curriculum. Am J Pharm Educ. 2009;73(8):156

20. WarholakTL, Queiruga $\mathrm{C}$, Roush R, Phan H. Medication error identification rates by pharmacy, medical and nursing students. Am J Pharm Educ. 2011;75(2):24.

21. Armor BL, Bulkley CF, Truong T, Carter SM. Assessing student pharmacists' ability to identify drug-related problems in patients with in a patient-centered medical home. Am J Pharm Educ. 2014;78(1):6.

22. Pham A. Improving Pharmacy Students' Education Through Enhanced Experiential Learning. Am J Pharm Educ. 2009;73(3):56.

23. Vinluan CM, Jabalie MM, Navarrete JP, Padilla ME. Evaluating the Types of Pharmacy Student Interventions Made During an Interprofessional 6-Week Adult Internal Medicine Rotation. J Pharm Pract. 2018;31(3):298-303.

24. Langebrake $\mathrm{C}$, Hilgarth $\mathrm{H}$. Clinical pharmacists' interventions in a German University Hospital. Pharm World Sci. 2010;32(2):194-9.

25. Zaman HH, Fun WH. Drug related problems in type 2 diabetes patients with hypertension: A cross-sectional retrospective study. BMC Endocr Disord. 2013;13(2):1-12.

26. Shareef J, Sandeep B, Shastry CS. Assessment of Drug Related Problems in Patients with Cardiovascular Diseases in a Tertiary Care Teaching Hospital. J Pharm Care. 2014;2(2):70-6.

27. Elnaem $\mathrm{MH}$, Bin $\mathrm{CIMZ}$, Abdul RNAH, Binti MNH, Binti SNM, et al. Knowledge and perceptions toward cardiology pharmacy education and training: Malaysian pharmacy students' perspectives. Curr Pharm Teach Learn. 2018;10(4):453-62.

28. Al-Hajje AH, Atoui F, Awada S, Rachidi S, Zein S, Salameh P. Drug-related problems identified by clinical pharmacist's students and pharmacist's interventions. Ann Pharm Fr. 2012;70(3):169-76.

29. Viktil KK, Blix HS, MogerTA, Reikvam A. Polypharmacy as commonly defined is an indicator of limited value in the assessment of drug-related problems. $\mathrm{Br} \mathrm{J}$ Clin Pharmacol. 2007;63(2):187-95.

30. Zaman HH, Chai LL. Drug-related problems in type 2 diabetes mellitus patients with dyslipidemia. BMC Public Health. 2013;13(1).

31. Abraham RR. Drug Related Problems and Reactive Pharmacist Interventions for Inpatients Receiving Cardiovascular Drugs. Int J Basic Med Sci Pharm. 2013;3(2):42-8.

Article History: Submission Date : 19-08-2018; Revised Date : 18-09-2018; Acceptance Date : 07-11-2018

Cite this article: Elnaem MH, Nazar NIM, Rahman NSA, Jamshed S. Analysis of Drug-Related Problems Identified by Future Pharmacists in Medical Wards of Tertiary Hospitals in the State of Pahang, Malaysia. JYoung Pharm. 2019;11(1):77-81. 